\section{Presentación inusual de cavidad ósea de Stafne: estudio mediante tomografía computarizada y resonancia magnética}

\section{Unusual Stafne's osseous cavity presentation: computerized tomography and magnetic resonance imaging study}

\begin{abstract}
Resumen
La cavidad ósea de Stafne (CS) ha sido descrita como una radiolucencia oval, de bordes definidos y corticalizados, localizada por debajo del conducto mandibular entre el primer molar y el ángulo de la mandíbula. Casos atípicos de presentación de la cavidad en forma lobulada con márgenes irregulares, escleróticos o incompletos, así como una localización inusual requieren el empleo de métodos imagenológicos que posibiliten realizar un diagnóstico diferencial, evitando un procedimiento invasivo. El objetivo del presente trabajo fue describir un caso de CS en un paciente masculino de 74 años de edad, con antecedente de cáncer de próstata. Las imágenes de tomografía computarizada de haz cónico evidenciaron una cavidad abierta hacia la tabla lingual por debajo del conducto mandibular. Las imágenes de resonancia magnética y tomografía computarizada multicorte permitieron identificar el contenido del defecto, identificado en ambos métodos imagenólogicos como tejido adiposo. El examen radiográfico de una CS de aspecto atípico debe ser complementado con estudios tomográficos y de resonancia magnética, estos proporcionan información relevante para el diagnóstico definitivo, limitando la realización de una exploración quirúrgica. En el caso clínico presentado, la caracterización de la extensión del defecto, su relación con los dientes y estructuras vecinas, así como la identificación del contenido permitieron descartar la presencia de una metástasis de cáncer de próstata.
\end{abstract}

Palabras clave: Mandíbula; Tomografía computarizada; Resonancia magnética (fuente: DeCS Bireme).

\begin{abstract}
The Stafne bone cavity (SC) has been described as an oval radiolucence, with defined and corticalized edges located below the jaw duct between the first molar and the angle of the jaw. Atypical cases of presentation of the cavity in lobed form with irregular, sclerotic or incomplete margins, as well as an unusual location require the use of imaging methods that make possible a differential diagnosis, avoiding an invasive procedure. The purpose of this work was to describe a case of SC in a 74-year-old male patient, with a
\end{abstract}

\section{Caso Clínico}

Adalsa Hernández-Andara ${ }^{1, a}$, Ana Isabel Ortega-Pertuz ${ }^{2, b}$, Juan Saavedra ${ }^{3, c}$, Marcos Gómez ${ }^{4, d}$, Mariana Villarroel-Dorrego $5, e$

${ }^{1}$ Clínica Félix Boada, Unidad de Imagen Dentomaxilofacial. Caracas, Venezuela.

${ }^{2}$ Universidad del Zulia, Facultad de Odontología, Instituto de Investigaciones, Área de Odontología Forense. Maracaibo, Venezuela.

${ }^{3}$ Centro de Especialidades Odontológicas. Chuao, Caracas, Venezuela.

${ }^{4}$ Instituto Venezolano de Investigaciones Científicas. Estado Miranda, Venezuela. ${ }^{5}$ Universidad Central de Venezuela, Facultad de Odontología, Instituto de Investigaciones Raúl Vicentelli. Caracas, Venezuela.

a Doctora en Diagnóstico Bucal.

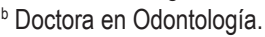

${ }^{c}$ Especialista en Endodoncia.

d Especialista en Cirugía Bucal y Maxilofacial.

e Doctora en Ciencias Odontológicas.

\section{Correspondencia:}

Adalsa Hernández-Andara

Correo electrónico: adalsa1@yahoo.com

Unidad de Imagen Dentomaxilofacial, Clínica Félix

Boada, Avenida Bolívar, Baruta, Caracas, Venezuela.

\section{Coautores:}

Ana Isabel Ortega-Pertuz

anitaortegav@gmail.com

Juan Saavedra

proendo@gmail.com

Marcos Gómez

marcosgomezmaxilofacial@gmail.com

Mariana Villarroel-Dorrego

villarroeldorrego@gmail.com

\section{Editora:}

Marieta Petkova-Gueorguieva

Universidad Nacional Mayor de San Marcos, Perú.

Conflicto de intereses: los autores declaran no tener conflicto de interés.

\section{Fuente de financiamiento: autofinanciado.}

Recibido: $17 / 07 / 19$

Aceptado: 30/09/19

Publicado: 27/11/19

(c) Los autores. Este artículo es publicado por la revista Odontología Sanmarquina de la Facultad de Odontología, Universidad Nacional Mayor de San Marcos. Este es un artículo de acceso abierto, distribuido bajo los términos de la licencia Creative Commons Atribucion - No Comercia_Compartir Igual 4.0 Internacional. (http://creativecommons.org/licenses/by-nc-sa/4.0/) que permite el uso no comercial, distribución y reproducción en cualquier medio, siempre que la obra original sea debidamente citada. 
history of prostate cancer. Cone beam computed tomography images showed an open cavity toward the lingual table below the mandibular canal. Magnetic resonance imaging and multi-cut computerized tomography allowed identifying the defect content, found adipose tissue. The radiographic examination of an atypical SC should be complemented with tomographic and magnetic resonance studies; these provide relevant information to the definitive diagnosis, limiting the performance of a surgical examination. In the clinical case presented, the characterization of the extension of the defect, its relationship with neighboring teeth and structures, as well as the identification of the content allowed us to rule out the presence of a prostate cancer metastasis.

Keywords: Mandible; Computed tomography; Magnetic resonance imaging (source: MeSH NLM).

\section{Introducción}

La cavidad ósea de Stafne (CS) fue reportada en 1942 como una radiolucencia bien definida, de bordes corticalizados, de forma oval o redondeada, asintomática, localizada comúnmente entre el ángulo de la mandíbula y el primer molar por debajo del canal mandibular ${ }^{1}$. La condición tiene una incidencia entre 0,10 a $0,40 \%$ en radiografías, sin embargo, este valor puede alcanzar el $6,06 \%$ en mandíbulas de cadáveres ${ }^{2-4}$. La CS es más frecuente en hombres, con una proporción hombre/mujer de $6: 1^{2,3,5}$, en edades comprendidas entre los 50 y 70 años ${ }^{3,4,6-9}$.

Aunque ocurre con más frecuencia en la región posterior de la mandíbula (variante posterior), la CS también puede aparecer en la zona anterior (variante anterior), en estos casos el defecto se observa entre el canino y el primer molar, por encima del músculo milohioideo y por debajo de los ápices radiculares si los dientes están presentes ${ }^{10,11}$. La cavidad ha sido reportada en la rama ascendente (variante de la rama ascendente) posterior al agujero lingual o en su cara externa. Usualmente la CS es unilateral, pero se han reportado casos bilaterales y de localización múltiple ${ }^{5,8,10,12,13}$.

Se han utilizado distintos sinónimos para describir la cavidad, entre ellos: quiste óseo de Stafne, cavidad ósea de Stafne, quiste óseo latente, defecto de desarrollo o idiopático de la mandíbula, depresión mandibular lingual de la glándula salival, defecto aberrante de la glándula salival, defecto óseo de la cortical lingual mandibular o una combinación de éstos ${ }^{2-5,9,11,14}$.

Típicamente la CS es un hallazgo radiográfico incidental, y a menudo tiene la apariencia de una radiolucencia unilocular con un diámetro entre $1 \mathrm{a} 3 \mathrm{~cm}$, forma oval, de bordes definidos y corticalizados, semejante a un quiste ${ }^{1,7,9,10,15-17}$. En ocasiones el borde se presenta esclerótico o no está bien definido ${ }^{8}$. Los casos multiloculares son infrecuentes ${ }^{13}$, y se ha reportado el adelgazamiento y expansión de la cortical vestibular, así como la solución de continuidad de la cortical inferior de la mandíbula ${ }^{7,9}$.

La etiología de la entidad todavía es incierta. Se ha considerado como principal causa del defecto la presión mecánica producida por el tejido glandular salival en la cortical lingual de la mandíbula ${ }^{10,15,16}$. Sin embargo, en defectos no asociados a una glándula salival, se ha seña- lado la posibilidad de erosión de la cortical causada por una lesión vascular adquirida o un lipoma ${ }^{4,7,15,18}$.

La identificación inicial de la cavidad es regularmente realizada en imágenes en $2 \mathrm{D}$, como una radiografía panorámica; en los casos de CS lobulada y márgenes irregulares, escleróticos o incompletos, así como una localización inusual, se debe recurrir a estudios adicionales para confirmar el diagnóstico como la tomografía computarizada de haz cónico (TCHC) ${ }^{4,9,13}$, tomografía computarizada multicorte (TCM) ${ }^{3,6-12,14-18,21-23}$ o resonancia magnética (RM) 4-7,13,15,17,20,22,23.

La CS ha sido clasificada de acuerdo a su aspecto en imágenes de TCM. Ariji et al. ${ }^{14}$ proponen una clasificación de acuerdo a la profundidad y contenido del defecto. Con respeto a la profundidad, el autor lo categoriza en: a) Tipo I: la cavidad está limitada a la porción medular de la mandíbula; b) Tipo II: el defecto alcanza la cortical vestibular de la mandíbula, pero no causa su expansión; c) Tipo III: la cavidad alcanza la cortical vestibular y causa su expansión. En cuanto a su contenido, se considera un Tipo F, cuando el contenido es tejido adiposo, el Tipo $S$ presenta tejido blando (nódulo linfático, vasos sanguíneos, tejido conectivo etc.) y el Tipo G constituye un defecto que contiene parte de una glándula salival. Minowa et al. ${ }^{15}$ clasifican la CS considerando la forma que presenta en vistas axiales de TCM, a saber: tipo amplio, tipo estrecho, tipo "margen liso" y tipo "margen irregular". Por su parte, Liu et al. 9 clasificaron el aspecto de la cavidad en imágenes de TCHC en cuanto a su relación con el conducto mandibular en: a) Separado: describe una cavidad que no contacta dicho conducto; b) Contacto: cuando la CS contacta el conducto mandibular y c) Extendido: en el caso que el defecto se extiende en el conducto. El autor también categoriza la relación de la CS con respecto a la cortical inferior de la mandibular en tres categorías: separado, en contacto y extendido, de forma similar a lo propuesto para el conducto mandibular.

El tratamiento de la CS no es necesario en vista que es considerada una variante anatómica y no una condición patológica. En general, el manejo de la entidad es el seguimiento radiológico conservador, de manera que la exploración quirúrgica seguida de biopsia se realiza cuando existen dudas en cuanto al diagnóstico y se sospecha la posibilidad de una patología ${ }^{4-6}$. Considerando lo anteriormente expuesto, el objetivo de este trabajo es 
describir un caso de variante posterior de CS con presentación inusual destacando el diagnóstico diferencial realizado mediante TCHC, TCM y RM.

\section{Reporte del caso}

Paciente masculino de 74 años, con antecedentes de cáncer $(\mathrm{CA})$ de próstata y tratado con prostactectomía hace 20 ańos, referido para evaluación, diagnóstico y tratamiento del diente 36. En la consulta relata dolor a la palpación a nivel apical vestibular y durante la masticación. Clínicamente el molar presentaba una restauración provisional de acrílico, con recidiva de caries en cervical y lingual. En las radiografías periapicales se observaba lo anteriormente descrito (Figura 1A), así como un abordaje endodóntico de emergencia y presencia de imagen radiolúcida en la zona apical. El diente fue intervenido en dos citas, con medicación intraconducto a base de $\mathrm{Ca}(\mathrm{OH})$, (UltraCal, Ultrradent Products Inc, South Jordan, UT) entre las mismas, acompañado de analgésicos (Ibuprofeno, $800 \mathrm{mg}$ en presencia de dolor) y antibiótico (Amoxicilina-Acido clavulánico 875/125 mg cada 12 horas). Los conductos se instrumentaron con limas manuales y posteriormente fueron intervenidos con instrumentos reciprocantes. El tratamiento fue finalizado mediante la obturación de los conductos con gutapercha y cemento endodóntico (Topseal, Dentsply Maillefer, Tulsa, OK, USA) sellado coronal con perno de fibra de vidrio (Tenax Fiber Trans, Coltene, Altstaetten, Schwyz, Suiza) y la reconstrucción con cemento de resina (ParaCore, Coltene, Altstaetten, Schwyz, Suiza) (Figura 1B). Actualmente se encuentra en fase de rehabilitación protésica.

Durante el examen preoperatorio del diente mediante TCHC (Carestream 9300, Atlanta, GA, USA) con un FOV (Fiel of view, por sus siglas en el inglés) de $9,5 \mathrm{~cm}$ y 0,180 $\mathrm{mm}$ de espesor del corte, se observó una imagen hipodensa por debajo del conducto mandibular, la cual no tenía relación con el diente de acuerdo a las vistas obtenidas (Figuras 2A, 2B, 2C). El paciente fue encaminado a una unidad de imagenología especializada para ampliar el estudio y descartar entre la presencia de una neoplasia debido a los antecedentes médicos del paciente o una variante anatómica.

Inicialmente se obtuvieron imágenes de RM (Ingenia, Philip Medical Systems, Andover MASS, USA) en secuencias de pulso T1 (Tiempo de relajación longitudinal, Tiempo Eco-TE 8 milisegundos/Tiempo de Repetición-TR 200 milisegundos) y T2 (Tiempo de relajación transversal, TE 80 milisegundos, TR 3837 milisegundos) las cuales mostraron en el plano axial, imagen hiperintensa sugestiva de presencia de tejido graso en el defecto antes mencionado (Figuras $3 \mathrm{~A}$ y 3B). Seguidamente, para suprimir la grasa fue realizada una ponderación en STIR (Short Time Inversion Recovery por sus siglas en inglés, TE 60 milisegundos /TR 2020 milisegundos, Tiempo de Inversión-TI 180), pero el material de restauración dentaria produjo artefactos que impidieron visualizar la zona referida (Figura 4). El estudio fue complementado con una TCM (Lightspeed VCT64, GE Medical Systems, Chicago, ILL, USA) de acuerdo a los siguientes parámetros técnico: FOV 26,2, filtro óseo, espesor del corte 0,625 , inclinación del gantry 0 , para determinar el coeficiente de atenuación en Unidades Housnfield (UH) del contenido de la cavidad.

En las reconstrucciones multiplanares y $3 \mathrm{D}$ mediante TCM (Figuras 5A y 5B), se observó una imagen hipodensa en la región apical del 36, sin compromiso del mismo, que se extendía hacia la base mandibular, adelgazándola, comprometiendo el conducto mandibular ipsilateral del cual no se observaba la pared lateral interna. En el plano axial (Figura 6) se evidenció una cavidad amplia abierta hacia lingual, de bordes irregulares, cuyo coeficiente de atenuación oscilaba entre -50 a -98 UH, sugestivo de contenido graso, lo cual fue confirmatorio de los hallazgos de la RM. No se verificó la presencia de glándula submandibular en el defecto.
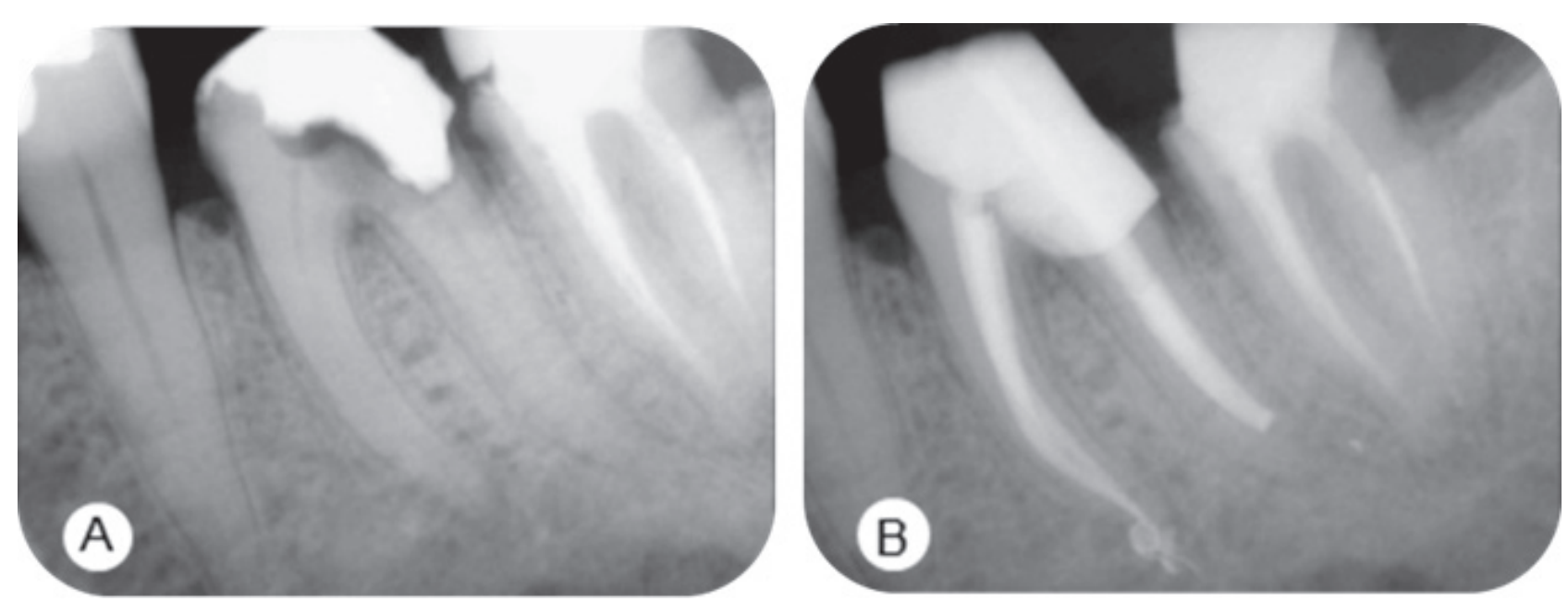

Figura 1. Radiografías periapicales digitales del diente 36. A. Radiografía preoperatoria donde se evidencia extensa imagen radiolúcida difusa en corona y tercio cervical radicular de la raíz distal, sugerente de recidiva de caries, nótese el compromiso de la región de furca y las imágenes radiolúcidas difusas en asociación a los ápices, sugestivas de lesión periapical. B. Radiografía postoperatoria mostrando la obturación de los conductos, acompañado de sellado coronal y reconstrucción para posterior rehabilitación protésica. 


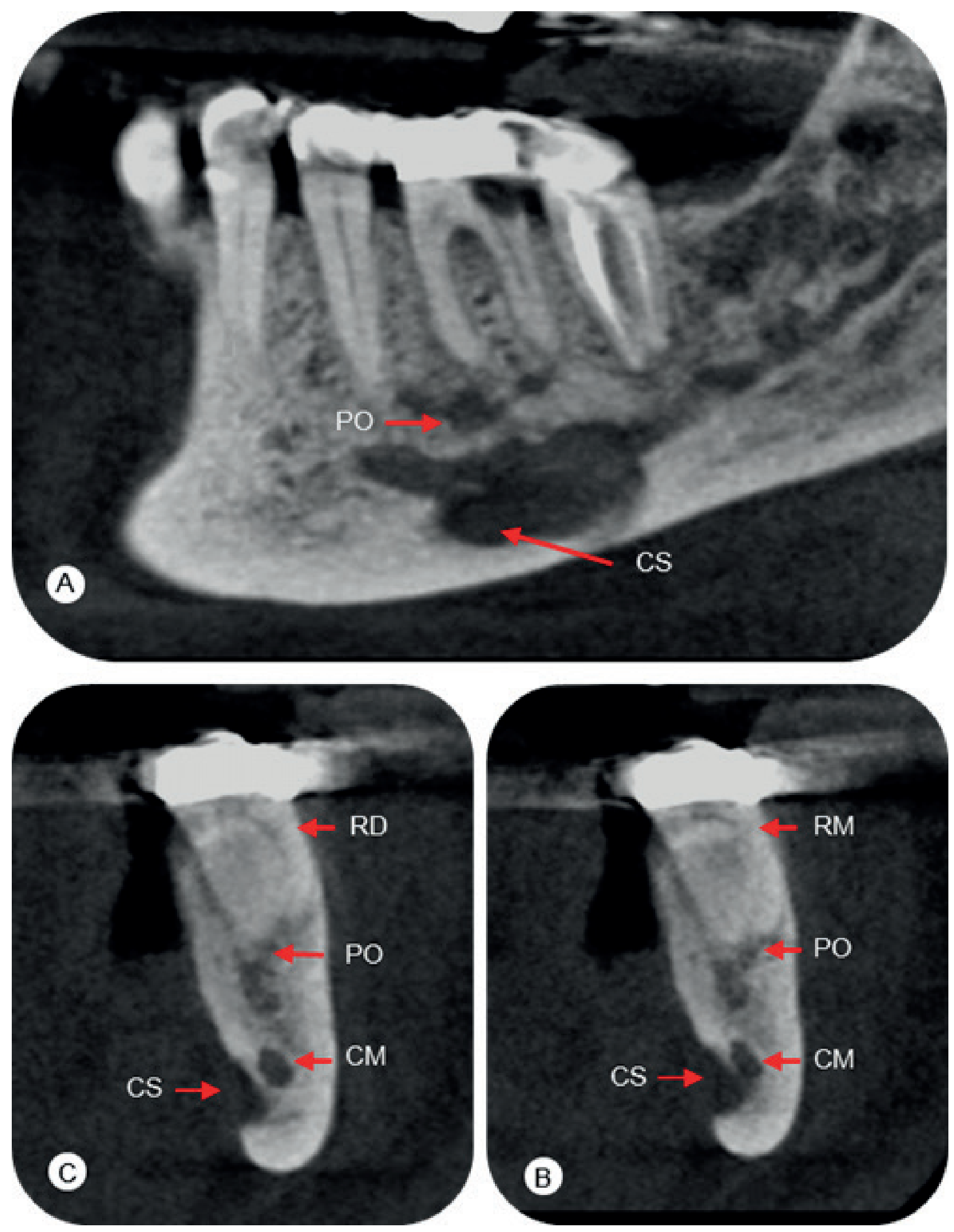

Figura 2. Imágenes adquiridas por tomografía computarizada de haz cónico. A. Vista sagital mostrando imagen hipodensa difusa en proximidad a la región apical del 36 sugerente de proceso osteolítico e imagen hipodensa por debajo del conducto mandibular, ovalada de bordes definidos con compromiso de conducto mandibular sugestiva de defecto óseo. B. Vista transaxial de raíz mesial del 36 donde se observa compromiso del conducto mandibular. C. Vista transaxial de distal del 36, con imagen apical hipodensa sugestiva de lesión periapical, e imagen hipodensa cavitaria próxima al conducto mandibular, que se abre hacia tabla ósea lingual

PO: proceso osteolítico; CS: cavidad ósea de Stafne; RM: raíz mesial del 36; RD: raíz distal del 36; CM: conducto mandibular. 

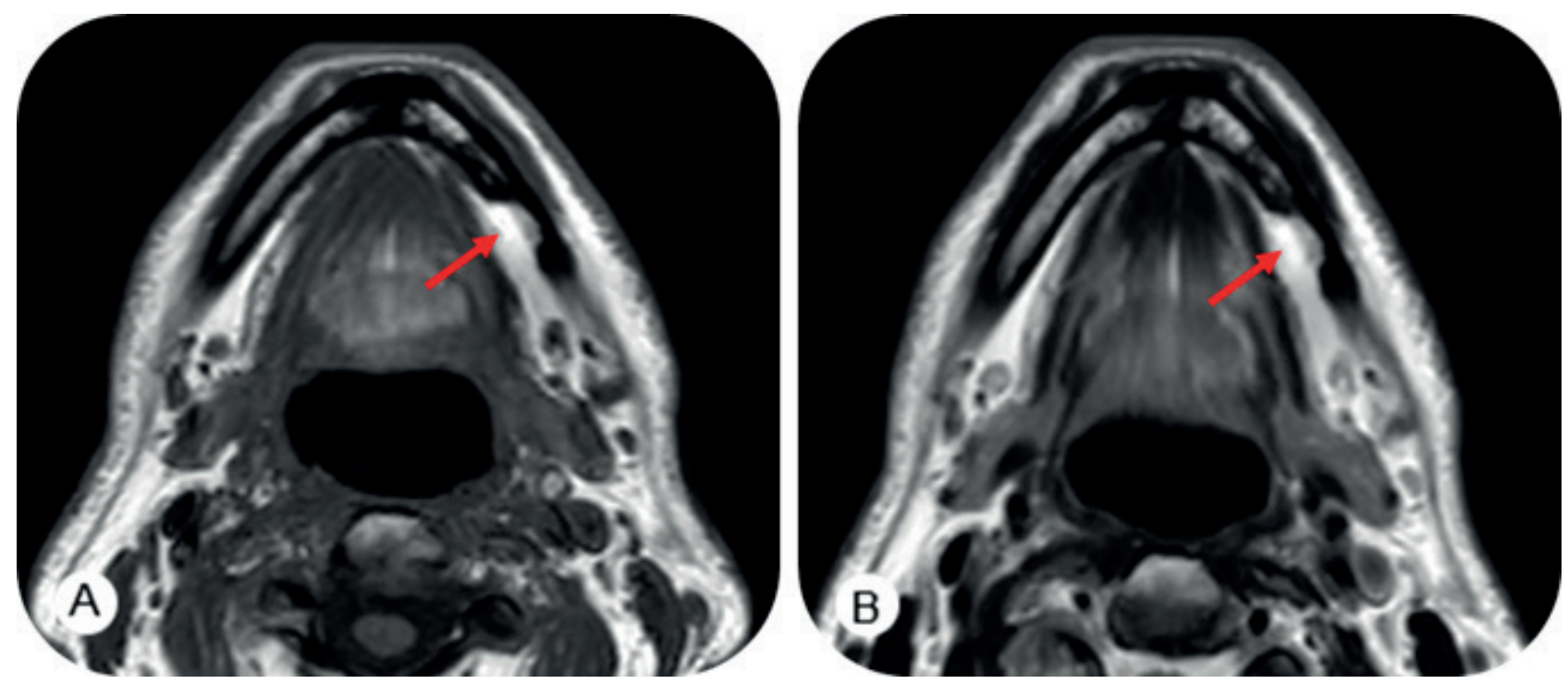

Figura 3. Imágenes adquiridas por resonancia magnética: A. Imagen axial ponderada en T1, donde se observa imagen de señal hiperintensa (flecha) sugestiva de tejido graso, ocupando imagen cavitaria que se abre hacia lingual. B. Imagen axial ponderada en $\mathrm{T} 2$, confirmando presencia de tejido graso (flecha).

T1: tiempo de relajación longitudinal; T2: tiempo de relajación transversal.

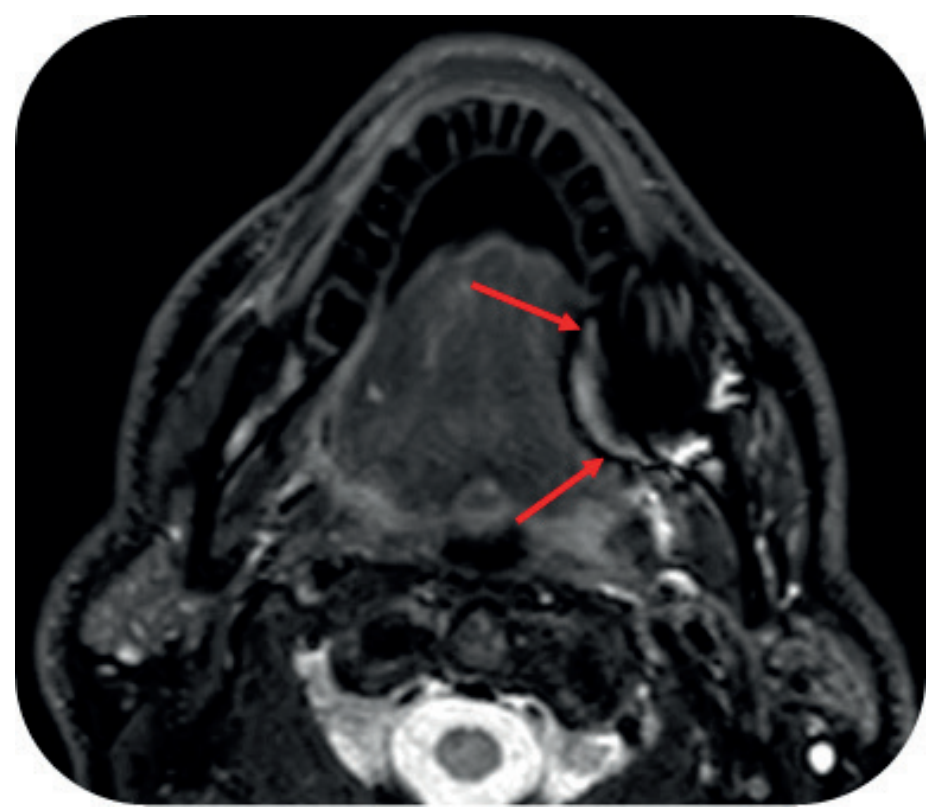

Figura 4. Imagen axial de resonancia magnética ponderada en STIR para supresión grasa, con presencia de artefacto (flechas) por restauración en el molar.

STIR: Short Time Inversion Recovery por sus siglas en inglés.

Se concluyó que las características imagenológicas fueron sugestivas de anomalía de cara interna de cuerpo mandibular, correspondiente a CS con contenido de tipo adiposo, que de acuerdo a la clasificación de Ariji et al. ${ }^{14}$, es del Tipo I y F. Debido a que la CS no requiere tratamiento, el paciente se encuentra en control radiográfico a criterio del clínico tratante.

\section{Discusión}

La CS posterior es la variante más frecuente, reportándose una prevalencia de 0,10 a $0,40 \%{ }^{2-4}$ y ha demostrado una mayor incidencia en hombres entre la quinta y séptima década de vida ${ }^{3,6-9,18}$. En este sentido, la cavidad descrita en el presente estudio, se observó en un individuo de sexo masculino de 74 ańos de edad, la misma se encontraba en la zona molar inferior, concordando con lo relatado en la literatura ${ }^{2-9}$.

La apariencia radiográfica de la CS generalmente, se ajusta a la descripción realizada por Stafne ${ }^{1}$, de una imagen radiolúcida oval de márgenes definidos y corticalizados localizada en la zona posterior de la mandíbula por debajo del conducto mandibular. En el paciente del caso descrito, la imagen mostraba un aspecto radiolúcido de bordes irregulares que compromete la pared lateral del 



Figura 5. Imágenes adquiridas por tomografía computarizada multicorte: A. Reconstrucción en 3D mostrando cavidad abierta hacia lingual (flecha) que compromete el conducto mandibular. B. En vista coronal se observa irregularidad de los bordes óseos (flecha) inusual en este tipo de variante anatómica y coeficiente de atenuación en Unidades Hounsfield de -93,1 (círculo rojo) que sugiere contenido graso. Asterisco en verde indica el ROI (region of interest por su siglas en ingles) para obtener el coeficiente de atenuación

conducto mandibular, lo cual es atípico en una CS, aunque Minowa et al. ${ }^{15}$ consideren dentro de su clasificación un tipo de defecto con "margen irregular". La apariencia osteolítica, la localización basal de la cavidad y el antecedente de CA de próstata del paciente hizo sospechar la presencia de una metástasis, debido también a que este tipo de lesiones puede observarse como una radiolucencia difusa, de bordes mal definidos y aspecto osteolítico ${ }^{24,25}$, semejante a lo observado en el caso. La TCHC de FOV reducido, indicada inicialmente para la valoración del diente 36, permitió conocer la extensión de la cavidad, el aspecto de sus bordes y la relación de la misma con las estructuras vecinas adyacentes, en coincidencia con lo relatado en otros estudios ${ }^{4,9,13}$, sin embargo, debido a las limitaciones propias de este recurso de imagen, en cuanto a la falta de contraste para el tejido blando, no pudo conocerse la naturaleza del contenido. Ante la duda de si se trataba o no de una patología se procedió a realizar una RM.

La RM constituye un método de diagnóstico no invasivo debido a que no emplea radiación inonizante y es empleada en los casos de CS para caracterizar y discriminar el tejido presente en el defecto a través de la utilización de distintas secuencias de pulso, sin requerir 


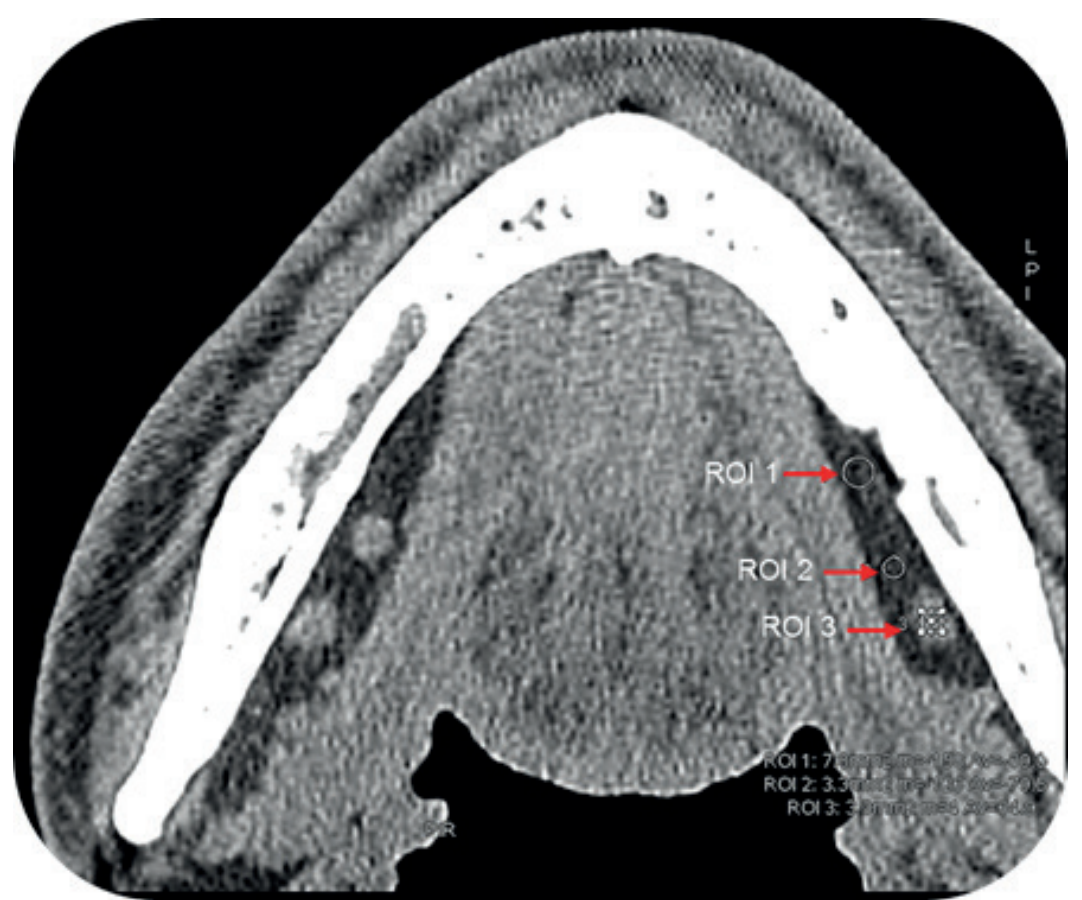

Figura 6. Imagen adquirida por tomografía computarizada multicorte. Vista axial mostrando el defecto óseo. Los círculos y asterisco muestran los ROI (región of interest, por sus siglas en inglés) para obtener los coeficientes de atenuación en Unidades Hounsfield, ROI 1 (intracavitario)=-57,6 sugestivo de componente graso, ROI 2 (tejido adyacente)=-98,5, ROI $3=49,8$ indicando imagen sugestiva de nódulo linfático

medios de contraste ${ }^{4,5,18,20-23}$. En el caso de CS mostrado, la secuencia T1 evidenció una imagen hiperintensa sugestiva de tejido graso, la cual disminuía su intensidad de señal en T2. Las imágenes ponderadas en STIR, usadas para suprimir grasa fueron imposibles de obtener debido a la presencia de artefactos en la imagen, proveniente de restauraciones dentarias próximas a la zona en estudio ${ }^{4}$. En vista de ello, se procedió a realizar una TCM, la cual posibilitó identificar el origen periférico de la entidad y la discontinuidad de la cortical lingual, lo que es esencial para descartar patologías ${ }^{3,22}$, en este caso las UH también fueron útiles en la identificación del contenido de la cavidad, como fue reportado por Aguiar et al. ${ }^{10}$ y Sisman et al. ${ }^{4}$, obteniéndose valores negativos en el coeficiente de atenuación indicativos de la presencia de tejido graso. De esta manera, el conjunto de exámenes volumétricos permitió formular el diagnóstico definitivo de CS, en concordancia con lo reportado en la literatura, evitando una biopsia excisional ${ }^{4,6}$ ante la sospecha de una metástasis de CA de próstata.

En contraste, Gómez-Da Silva et al. ${ }^{21}$ relataron la presencia de una CS en un paciente con CA de próstata y metástasis múltiples, con una apariencia diferente a la del presente caso. La radiografía panorámica mostraba una imagen radiolúcida unilocular, de bordes escleróticos, en la zona posterior derecha de la mandíbula, mientras que la TCM reveló la presencia de una imagen hipodensa, bien delimitada en la cortical lingual. Si bien la sospecha inicial era de una metástasis en mandíbula, las características imagenológicas, en conjunto con la falta de síntomas asociados y un examen óseo con radionucleótidos en el que no se evidenció la hipercaptación del fármaco en los maxilares, condujo al diagnóstico de CS.
En relación a contenido de la CS, la literatura ha reportado que generalmente la cavidad presenta en su interior, tejido glandular salival de acuerdo a su localización 2,11,14,19 sin embargo, puede incluir tejido conectivo fibroso, adiposo, muscular, nervios, nódulos linfáticos y vasos sanguíneos, o estar vacío ${ }^{11,13,14,18,19}$. Estos hallazgos podrían estar asociados a la remoción quirúrgica de tejido blando adyacente al defecto, así como a herniación intermitente de la glándula adyacente o regresión de dicha herniación ${ }^{6,10,19,20}$. Se ha señalado que la presencia de grasa en la cavidad pudiera estar relacionada con un lipoma ${ }^{15}$, en este caso se comprobó mediante las imágenes de RM que se trataba de una extensión del tejido adiposo de la región submandibular en la zona.

El diagnóstico diferencial de la CS ha incluido tumores benignos de las glándulas salivales, tumores neurogénicos, hemangiomas, mixoma, lesión central de células gigantes, hiperparatiroidismo, quistes odontogénicos, quiste óseo simple, quiste óseo aneurismático, ameloblastoma, lesiones fibro-óseas, mieloma múltiple, granuloma eosinofílico, fistula arteriovenosa y enfermedad metastásica $4,8,19,20$. En el presente caso, aunque el aspecto ostelítico de la imagen orientaba la impresión diagnóstica inicial de una patología, la remisión de la sintomatología dolorosa después del tratamiento endodóntico y el hallazgo de tejido adiposo en el interior de la cavidad condujeron al diagnóstico de una CS, indicando por ello, el seguimiento radiográfico.

Es importante considerar que las metástasis de CA de próstata y otros tumores malignos en los maxilares suelen cursar con aumento de volumen que conduce a asimetría facial y regularmente están asociadas con sín- 
tomas como dolor, parestesia y destrucción ósea, sin embargo, en los casos que carecen de estas características, es difícil diferenciarlas de condiciones benignas o alteraciones óseas como la $\mathrm{CS}^{21}$, por ello la necesidad de una exploración imagenológica exhaustiva que permita orientar el diagnóstico.

En conclusión, el examen radiológico de una CS de aspecto atípico debe ser complementado con estudios tomográficos y de RM, ambos, dentro de sus limitaciones, proporcionan información relevante para el diagnóstico diferencial evitando procedimientos invasivos. En el caso clínico presentado, conocer la extensión del defecto, su relación con los dientes y estructuras vecinas, así como la identificación de tejido graso en su interior permitieron descartar la presencia de una metástasis de CA de próstata.

\section{Referencias bibliográficas}

1. Stafne EC. Bone cavities situated near the angle of the mandible. J Am Dent Assoc. 1942;29:1969-72.

2. Chen CY, Ohba T. Analysis of radiological findings of Stafne's idiopathic bone cavity. Dentomaxillofac Radiol. 1981;10(1):18-23.

3. Quesada-Gomez C, Valmaseda-Castellon E, Berini-Aytes L, Gay-Escoda C. Stafne Bone Cavity: A Retrospective Study of 11 Cases. Med Oral Patol Oral Cir Bucal. 2006;11(3):e277-e80.

4. Sisman Y, Miloglu O, Sekerci AE, Yilmaz AB, Demirtas O, Tokmak TT. Radiographic evaluation on prevalence of Stafne bone defect: A study from two centres in Turkey. Dentomaxillofac Radiol. 2012;41(2):15-8.

5. Philipsen HP, Takata T, Reichart PA, Sato S, Suei Y. Lingual and buccal mandibular bone depressions: A review based on 583 cases from a world-wide literature survey, including 69 new cases from Japan. Dentomaxillofac Radiol. 2002;31(5):281-90.

6. Segev Y, Puterman M, Bodner L. Stafne bone cavity Magnetic resonance imaging. Med Oral Patol Oral Cir Bucal 2006;11(4):E345-7.

7. Campos PSF, Oliveira JAC, Dantas JA, de Melo DP, Pena N, Santos LAN, Crusoé-Rebello IM. Stafne's defect wirh bucal cortical expansion: A case report. Int J Dent. 2010: 515931. DoiI:10.1155/2010/515931

8. Turkoglu K, Orhan K. Stafne bone cavity in the anterior mandible. J Craniofac Surg. 2010;21(6):1769-75.

9. Liu L, Kang BC, Yoon SJ, Lee SJ, Hwong SA. Radiographic features of lingual mandibular bone depression using dental cone beam computed tomography. Dentomaxillofac Radiol. 2018 Jul;47(6):20170383. DOI: $10.1259 / \mathrm{dmfr} .20170383$

10. Aguiar LB, Neves FS, Bastos LC, Crusoé-Rebello I, Ambrosano GM, Campos PS. Multiple Stafne bone defects: A rare entity. ISRN Dent. 2011;792145. DOI: $10.5402 / 2011 / 792145$

11. Dereci O, Duran S. Intraorally exposed anterior Stafne bone defect. A case report. Oral Surg Oral Pathol Oral Radiol. 2012;113(5):e1-e3.
12. Campos PSF, Panella J, Crusoé-Rebello IM, Azevedo RA, Pena N, Cunna T. Mandibular ramus-related Stafne's bone cavity. Dentomaxillofac Radiol. 2003;33(1):63-6.

13. Ozaki H, Ishikawa S, Kitabatake K, Yusa K, Tachibana $\mathrm{H}$, Iino $M$. A Case of simultaneous unilateral anterior and posterior Stafne bone defects. Case Rep Dent. 2015;983956. DOI:10.1155/2015/983956.

14. Ariji E, Fujiwara N, Tabata O, Nakayama E, Kanda S, Shiratsuchi Y, et al. Stafne's Bone cavity classification based on outline and content determined by computed tomography. Oral Surg Oral Med Oral Pathol. 1993;76(3):375-80.

15. Minowa K, Inoue N, Sawamura T, Matsuda A, Totsuka Y, Nakamura M. Evaluation of static bone cavities with CT and MRI. Dentomaxillofac Radiol. 2003;32(1):2-7.

16. Shimizu M, Osa N, Okamura K, Yoshiura K. CT Analysis of the Stafne's Bone defects of the mandible. Dentomaxillofacial Radiology. 2006;35(2):95-102.

17. Minowa K, Inoue N, Izommana Y, Ashikaga Y, Chu B, Maravilla KR et al. Static bone cavity of the mandible. Computed tomography findings with histopathologic correlations. Acta Radiol. 2006;47(7):705-9.

18. Sisman Y, Etöz OA, Mavili E, Sahman H, Ertas ET. Anterior Stafne defect mimicking a residual cyst. A case report. Dentomaxillofac Radiol. 2010;39(2):124-6.

19. Ertas ET, Atici MY, Kalabalik F, Ince O. Investigation and differential diagnosis of Stafne bone cavities with cone beam computed tomography and magnetic resonance imaging: Report of two cases. J Oral Maxillofac Radiol 2015;3(3):92-6.

20. Branstetter BF, Weissman JL, Kaplan SB. Imaging of a Stafne bone cavity: What MR adds and why a new name is needed. AJNR Am J Neuroradiol. 1999;20(4):587-9.

21. Gómez-Da Silva W, Kemp AT, Santos-Silva AR, Diz MPZ, Brandão TB. Stafne's bone defect in a metastatic prostate cancer patient. A diagnostic conundrum. J Clin Exp Dent. 2018;10(1):e88-91.

22. Hisatomi M, Munhoz L, Asaumi J, Arita ES. Lingual mandibular bone defect: imaging features in panoramic radiograph, multislice computed tomography and magnetic resonance imaging. Braz Dent Sci. 2018;21(2): 247-52.

23. He J, Wang J, Yongjie H, Liu W. Diagnosis and management of Stafne bone cavity with emphasis on unsual contents and location. J Dent Scie. DOI: https://doi. org/10.1016/j.jds.2019.06.001.

24. Menezes JDS, Capellari PFM, Capelari MM, Gonçalves PZ, Toledo GL; Toledo Filho JL, Sales-Peres A, Marzola C. Mandibular metastasis of adenocarcinoma from prostate cancer: case report according to epidemiology and current therapeutical trends of the advanced prostate cancer. J Appl Oral Sci. 2014;21(5):490-5.

25. D'Silva NJ, Summerlin DJ, Cordell KG, Abdelsayed RA, Tomich CE, Hanks CT, et al. Metastatic tumors in the jaws: A retrospective study of 114 cases. J Am Dent Assoc. 2006;137(2):1667-72. 